\title{
NON-LINEAR STRETCH MODULUS OF MATERIALS UNDER SIMPLE TENSION
}

\author{
Elli Gkouti $^{1^{*}}$, Burak Yenigun ${ }^{1}$, Krystof Jankowski $^{2}$, Aleksander Czekanski $^{1^{* *}}$ \\ ${ }^{1}$ Department of Mechanical Engineering, Lassonde School of Engineering, York University, Toronto, ON, Canada \\ ${ }^{2}$ Magna Closures of America Inc., Troy, MI, USA \\ *gkoutiel@yorku.ca,**alex.czekanski@lassonde.yorku.ca
}

\begin{abstract}
In isotropic elasticity concerning large deformations, the non-linear response of materials is described by parameters, which are not constants but scalar functions of deformations. The procedure of defining them is not simple as there are several ways of calculating the strain and stress tensors, based on the different configuration of non-linear deformation. In the current work, non-linear equations of stretch modulus are presented and their dependence on both stress and strain tensors is shown. For defining the strainenergy density function of the hyperelastic materials, selected experiments were done considering four materials: natural rubber, neoprene, EPDM and silicone. These materials were examined under simple tension and from the arising results, Cauchy (true stress) and 1st Piola-Kirchhoff (engineering) stress tensor are calculated, using an existing model of strainenergy function, which best fits the obtained experimental data. For each of the above stress tensors, the non-linear case of the stretch modulus is defined for the examined materials. The results were combined to each other for small and large deformations.
\end{abstract}

Keywords-rubber; stretch modulus; non-linear; hyperelastic; incompressible materials;

\section{INTRODUCTION}

In isotropic theory of elasticity, only two parameters are needed for describing the response of a material, which is subjected to a deformation. In the case of linear deformation, the couple of parameters are Poisson's ratio and Young's modulus [1], which are constant and are used for determining any other information, which describes the mechanical behavior. In contrast, for large deformations, these parameters are scalar functions of deformation, which constitutes a more complicated procedure of describing the mechanical response of a material. Namely, finite deformation includes different ways of expressing strain and stress, due to the several ways of describing deformations [2]. More specific, in order to describe the deformation of a material, in respect to its initial position or to its current point, 1st Piola-Kirchhoff and Cauchy stress tensors, respectively, must be defined. Moreover, the strain tensor must be compatible to the configuration, which the stress tensors are considered. In the present paper, the non-linear expressions of stretch modulus are defined for describing the change of Cauchy and 1st Piola-Kirchhoff stress tensors with the changes of the logarithmic strain and the displacement gradient, respectively [2]-[3]. In order to calculate the stress tensors for hyperelastic, incompressible materials, the strainenergy density function must be defined first, and this is possible by selecting one of the existing models of this function from the bibliography [4]. The choice of the most compatible strain-energy function model is a result of the best fitting of the experimental data obtained. Two different samples of four materials are examined in simple tension. The materials are: natural rubber, EPDM, neoprene and silicone. For each of them, Cauchy and 1st Piola-Kirchhoff stress tensors are defined, for the corresponding deformation. The results present difference between small and large deformations. Finally, the non-linear stretch modulus for each material is been calculated and compared with the classic Young's modulus of the linear theory.

\section{THEORETICAL BASIS}

\section{A. Strain and Stress Tensors}

Consider an incompressible, isotropic material, which is hyperelastic, namely the strain-energy density function $W=$ $W\left(I_{1}, I_{2}\right)$ remains unaffected for the deformation and so, it can be expressed as a function of the principal invariants $I_{1}=\lambda_{1}{ }^{2}+\lambda_{2}{ }^{2}+\lambda_{3}{ }^{2}$ and $I_{2}=\lambda_{1}{ }^{2} \lambda_{2}{ }^{2}+\lambda_{2}{ }^{2} \lambda_{3}{ }^{2}+\lambda_{3}{ }^{2} \lambda_{1}{ }^{2}$, where $\lambda_{k}(k=1,2,3)$ are the principal stretch ratios. In the current research, the polynomial model with two terms is selected, due to its best fit with the experimental data [4]. The selected strain-energy density function is described by

$$
\begin{aligned}
W= & C_{10}\left(I_{1}-3\right)+C_{01}\left(I_{2}-3\right)+C_{11}\left(I_{1}-3\right)\left(I_{2}-3\right) \\
& +C_{20}\left(I_{1}-3\right)^{2}+C_{02}\left(I_{2}-3\right)^{2},
\end{aligned}
$$

where $C_{i j}(i, j=0,1,2)$ are material constants. Several stress and strain tensors are used in finite deformation, depending on the considered configuration. For the present work, two stress tensors are defined, which are widely used in non-linear theory of elasticity, for incompressible materials. The force per unit area in the current configuration is represented by the Cauchy stress tensor, known, also as true stress, and takes the form 


$$
\boldsymbol{\sigma}=-p \mathbf{I}+b_{1} \mathbf{B}+b_{-1} \mathbf{B}^{-1}
$$

where $\mathbf{B}$ is the left Cauchy-Green deformation gradient, which has the squared stretches $\lambda_{\mathrm{i}}{ }^{2}$ as principal values; $\boldsymbol{I}$ is the identity tensor, $p$ is an unknown hydrostatic stress and $b_{1}=2 \partial \mathrm{W} / \partial I_{1}, b_{-1}=$ $-2 \partial \mathrm{W} / \partial I_{2}$, are two response functions depending on the stress invariants, determined by experiments [5]. The force per unit area in the reference configuration is presented by the 1st PiolaKirchhoff stress tensor, known as engineering stress, and is expressed by

$$
\mathbf{P}=\boldsymbol{\sigma} \boldsymbol{F}^{-T}
$$

where $\boldsymbol{\sigma}$ is the Cauchy stress tensor and $\boldsymbol{F}$ is the deformation gradient. Any other stress tensor can be defined as a function of the deformation gradient or the stress tensors calculated above, such as the symmetric 2nd Piola-Kirchhoff stress tensor, commonly used in computational analysis. For the present work, the case of a simple tension acting in the first direction is studied, where the corresponding extension, takes the form

$$
x_{1}=\lambda X_{1}, x_{2}=k(\lambda) X_{2}, x_{3}=k(\lambda) X_{3},
$$

where $\left(x_{1}, x_{2}, x_{3}\right)$ and $\left(X_{1}, X_{2}, X_{3}\right)$ are the Cartesian coordinates for the current and reference configuration, respectively; $\lambda$ is the extension ratio in the direction of where the force is applied, and $k(\lambda)$ the stretch ratio in the orthogonal direction. For the special case of isochoric deformation, namely $\operatorname{det} F=1$, the orthogonal stretch takes the form $k(\lambda)=\lambda^{-1 / 2}$.

\section{B. Non-Linear Stretch Modulus}

Correspondingly to the linear theory, the material's response to the extension must be described by considering both stress and strain tensors, which are functions of finite deformation. Materials get stiffer or softer when a loading is applied. The parameter describing the above tendency is known as the non-linear stretch modulus [2], [6] and [7], which has different forms according to the selected stress and strain tensors. The stretch modulus, for the case of the Cauchy stress tensor, is defined in terms of the logarithmic strain tensor $e_{0}=\ln (\lambda)$, in the form

$$
E_{C}(\lambda)=\frac{\sigma_{1}(\lambda)}{\ln (\lambda)}
$$

where $\sigma_{1}(\lambda)$ is the only non-zero component of the Cauchy stress tensor and $\lambda$ is the axial stretch ratio in the direction of the loading. For the case of the 1st Piola-Kirchhoff stress tensor, the stretch modulus, arising from the displacement gradient, is expressed here, in terms of the stretch ratio as

$$
E_{P}(\lambda)=\frac{P_{1}(\lambda)}{\lambda^{3 / 2}-1}\left(\lambda^{1 / 2}+\frac{1}{2 \lambda}\right),
$$

where $P_{1}(\lambda)$ is the only non-zero component of the 1 st PiolaKirchhoff stress tensor.

\section{EXPERIMENTAL RESULTS AND DISCUSSION}

For the present work, two samples of four different materials are subjected to simple tension until they have reached their fracture point, with $0.1 \mathrm{~mm} / \mathrm{s}$ speed, in room temperature. The arising test data was used for defining the reaction functions $b_{1}, b_{-1}$ needed for calculating the Cauchy and 1st Piola-Kirchhoff stress tensors, given by (2)-(3). The selected model of strain-energy density function, which best fits the experimental data, was Polynomial with two terms, given by (1). In Fig. 1 and Fig. 3, the only non-zero Cauchy and

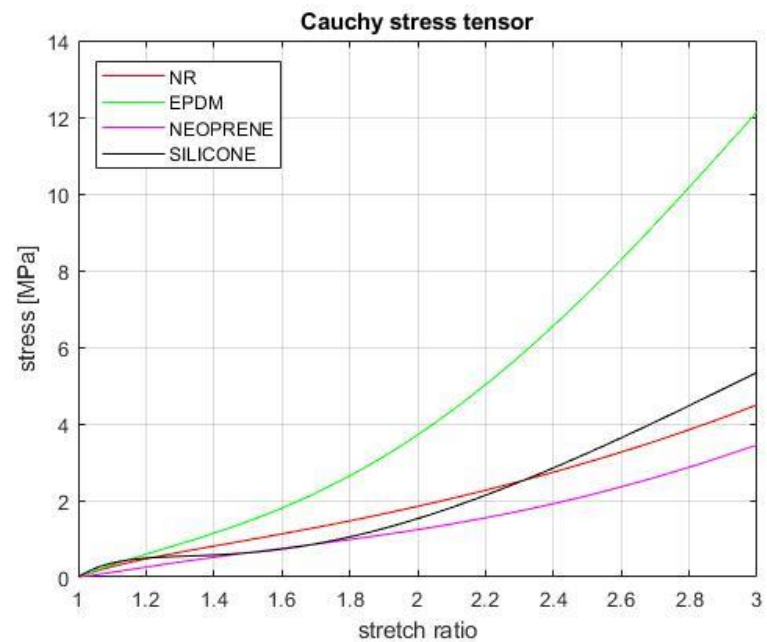

Figure 1. Cauchy stress tensor versus axial stretch ratio, for four different materials

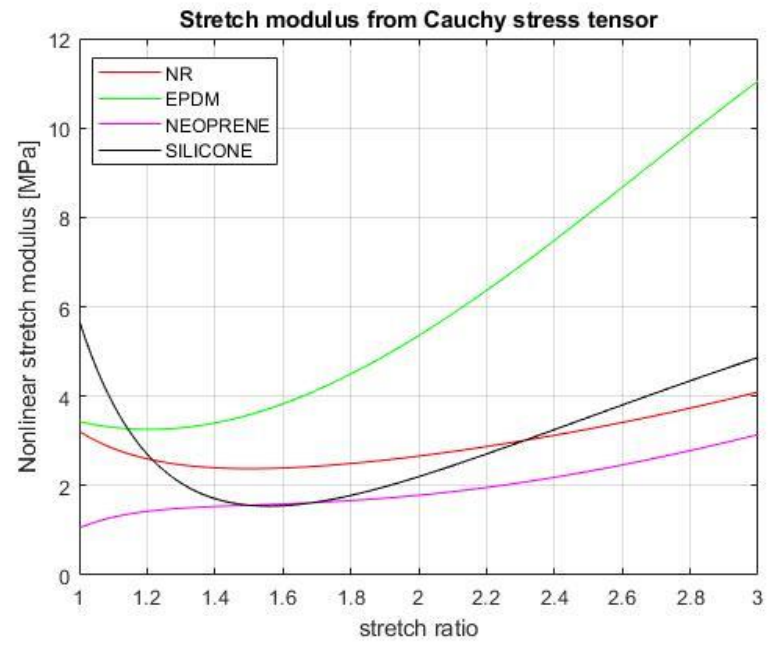

Figure 2. Non-linear stretch modulus for Cauchy stress tensor versus axial stretch ratio, for four different materials 
1st Piola-Kirchhoff stress components are shown for some of the examined materials, and based on the corresponding results, the nonlinear stretch modulus is calculated and shown, in Fig. 2 and Fig. 4. It is obvious that, all the materials, except for EPDM, have a similar behavior. For small deformation, the different stress tensors are almost equal and linear, and the stretch modulus tend to infinite as the denominator of the corresponding equations (5) and (6) tend to zero. For that case, the stretch limit $(\lambda \rightarrow 1)$ is selected for describing the change of stress to strain, coinciding with the constant Young's modulus, for each material.

\section{CONCLUSIONS}

Consequently, the material mechanical response to finite deformation can be defined by several ways, in respect to the selected configuration. Moreover, describing an axial stretch, in respect to any configuration of their position, the stress tensors can be calculated in several ways. For the force applied to the area of the current configuration, the Cauchy stress tensor is used, but for the force considered to the area of the reference position of the materials, the proper stress tensor is the 1st Piola-Kirchhoff stress tensor. For infinitesimal deformations, the change of stress in respect to the corresponding stretch ratio is almost linear and equal for each stress measurement. By selecting the most suitable configuration describing the material's response to deformation, the determination of a parameter concerning the stiffness (or softness) of a material, is possible. This parameter is a scalar function of deformation, known as the non-linear stretch modulus and for small deformation, it is equal to the Young's modulus of the linear theory.

\section{REFERENCES}

[1] A.E.H. Love, A Treatise on the Mathematical Theory of Elasticity, 4th ed., New York, NY: Dover, 1927.

[2] R.W. Ogden, Ogden, Non-Linear Elastic Deformation, 2nd ed., New York, NY: Dover, 1997.

[3] H. Hencky, "Uber die form des elastizitatsgesetzes bei ideal elastischen stiffen," Z. Tech. Phys., vol. 9, pp. 215-220, 1928.

[4] R.S. Rivlin and D.W. Saunders, "Large deformations of isotropic materials VII. Experiments on the deformation of rubber," Phi. Trans. Roy. Soc. London Series, vol. A243 (865), pp. 251-288, 1951.

[5] C. Truesdell and W. Noll, The Non-Linear Fields Theories of Mechanics, Berlin, Germany: Springer-Verlag, 1965.

[6] L.A. Mihai and A. Goriely, "How to characterize a nonlinear elastic material? A review on nonlinear constitutive parameters in isotropic finite elasticity," Proc. Roy. Soc. , vol. A473, pp. 20170607, 2017.

[7] M. Destrate, G. Saccomandi and I. Sgura, "Methodical fitting for mathematical models of rubber-like materials," Proc. Roy. Soc. , vol. A473, pp. 20160811, 2017.

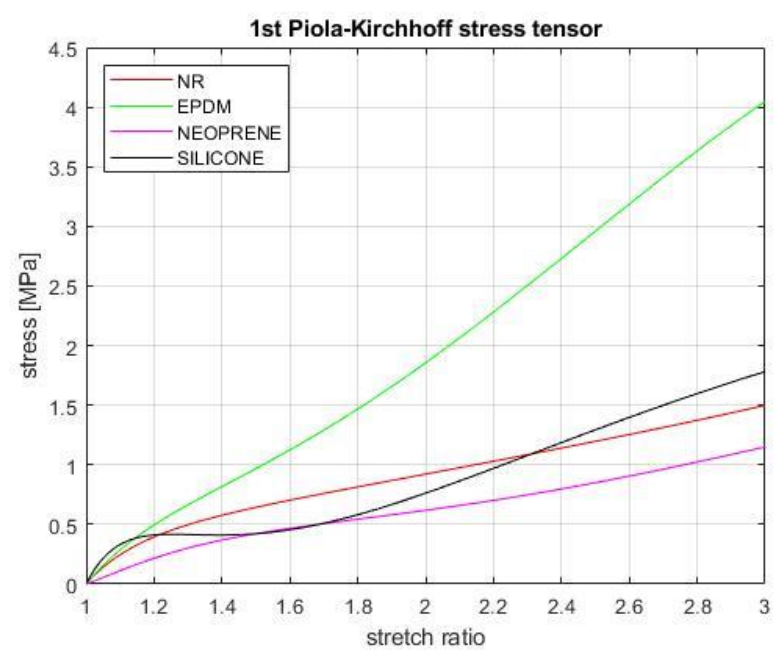

Figure 3. $1^{\text {st }}$ Piola-Kirchhoff stress tensor versus axial stretch ratio, for four different materials

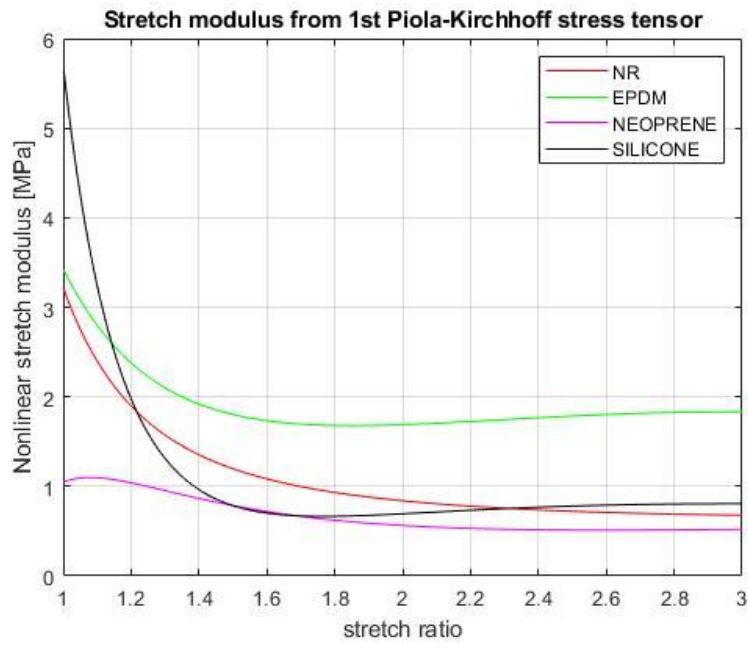

Figure 4. Non-linear stretch modulus for $1^{\text {st }}$ Piola-Kirchhoff stress tensor versus axial stretch ratio, for four different materials 